\title{
Protective effects of D-Limonene against transient cerebral ischemia in stroke-prone spontaneously hypertensive rats
}

\author{
XIFENG WANG, GANG LI and WEI SHEN \\ Department of Neurology, Puai Hospital of Tongji Medical College, Huazhong University \\ of Science and Technology, Wuhan, Hubei 430033, P.R. China
}

Received May 14, 2017; Accepted August 23, 2017

DOI: $10.3892 /$ etm.2017.5509

\begin{abstract}
Stroke is a leading cause of disability and death world-wide and there is currently a lack of effective treatments for acute stroke. D-Limonene is a common natural monocyclic monoterpene possessing various activities. The present study aimed to evaluate the therapeutic efficacy of D-limonene against ischemia-associated cerebral injury in hypertensive SHRsp rats. Although systolic blood pressure was not altered by ischemia, D-Limonene decreased the systolic blood pressure of SHRsp rats following stroke. Induction of stroke resulted in increased escape latency time, decreased time spent in the target quadrant in the probe trial, decreased capacity to distinguish between familiar objects and novel objects, and increased sensory neglect in the SHRsp rat, however these symptoms were significantly inhibited by D-limonene. D-limonene also decreased the cerebral infarct size in the SHRsp rats following stroke. D-Limonene markedly decreased the mRNA expression of interleukin-1 $\beta$, monocyte chemoattractant protein-1 and cyclooxygenase-2 in SHRsp rats following stroke. The mRNA expression of vascular endothelial growth factor in the brain of SHRsp rats following stroke was significantly increased by D-Limonene. D-Limonene increased the activities of superoxide dismutase and catalase, decreased the malondialdehyde level, increased glutathione content and reduced the DHE-staining in SHRsp rats following stroke. Overall, inhibition of cerebral inflammation, vascular remodeling and antioxidant activities of D-Limonene may be involved in the protective effects against ischemia-induced damage in SHRsp rats. The present study identified D-Limonene as a potential therapeutic candidate for treatment of stroke-associated cerebral and vascular damage under conditions of hypertension.
\end{abstract}

Correspondence to: Dr Wei Shen, Department of Neurology, Puai Hospital of Tongji Medical College, Huazhong University of Science and Technology, 76 Jiefang Avenue, Wuhan, Hubei 430033, P.R. China

E-mail: wenshen666@yeah.net

Key words: D-limonene, stroke, cerebral damage, inflammation, oxidative stress

\section{Introduction}

Stroke is a leading cause of disability and death world-wide and it is lack of effective treatments for acute stroke. Cerebral small vessel disease (CSVD) contributes to only 20-30\% of all strokes (1), and it substantially increases the risk of stroke in the future (2) and accounts for about $45 \%$ of dementia cases (3). Progressive CSVD and stroke lead to decline of cognition, causing a major burden on the individual and the healthcare system $(4,5)$. One in three U.S. adults-67 million people-is estimated to have high blood pressure, which if not controlled can result in CSVD and cause cerebral stroke (6). Stroke-prone spontaneously hypertensive (SHRsp) rats, derived from Wistar-Kyoto (WKY) rats, serve as a widely-used model of severe hypertension, given that the rats naturally progress to hypertension starting from 4 weeks to 12 weeks of age $(7,8)$ and more than $95 \%$ of rats die of stroke (9). In SHRsp rats, cerebral blood flow could be reduced by severe hypertension and monocyte adhesion to the cerebral artery endothelium can be increased (10). The increased blood pressure induces cerebral vasogenic oedema (11).

D-Limonene, a common natural monocyclic monoterpene, also known as 1-methyl-4-(1-methyl ethenyl) cyclohexene, which is widely distributed as a natural nonnutritive constituent in a variety of foods, including fruits (citrus fruits, especially lemon and orange) (12), vegetables (carrots) (13), coffee, beverages, meat, and spices (nutmeg) (14). It is found naturally in orange juice at an average concentration of $100 \mathrm{mg} / \mathrm{l}(15)$. D-Limonene is usually used as a lemon fragrance in soaps, lotions, creams, detergents, and perfumes and as a flavoring agent in foods, beverages, and chewing gum (15). In the last years, numerous studies have found that D-limonene possesses powerful antioxidative properties and protects organisms from oxidative damage $(16,17)$. In addition, D-limonene exhibits antitumorigenic, hepatoprotective, immunomodulatory, and anti-inflammatory properties (18-22). In particular, D-limonene has been shown to attenuate stress-induced hypertension and stress responses in SHRsp rats $(23,24)$. However, the effect of D-limonene on cognitive and memory function and ischemic injury in SHRsp rats is not clear.

The present study aimed to evaluate the therapeutic efficacy of D-limonene against the ischemia-resulted injury of cognitive and memory and behavioral function and vascular remodeling. We showed that D-limonene substantially 
attenuated the decline of cognitive and memory and behavioral function and ischemic injury in SHRsp rats.

\section{Materials and methods}

Animals and treatment. WKY and SHRsp rats were purchased from SLK (Shanghai, China) and then maintained in rooms under conditions of controlled temperature $\left(21 \pm 2^{\circ} \mathrm{C}\right)$, humidity $(60 \pm 10 \%)$, and a $12 \mathrm{~h} \mathrm{light/dark} \mathrm{cycle} \mathrm{(exposed} \mathrm{to} \mathrm{artificial}$ light from 7:00 am to 7:00 pm) and air ventilation $(15 \mathrm{~min} / \mathrm{h})$. The rats were fed with a standard rodent laboratory diet and water ad libitum. Adequate measures were taken to minimize pain or discomfort, and the experiments were carried out in accordance with the conventional guidelines for experimentation with animals (National Institute of Health, Publication no. 85-23, revised 1996). All animal procedures were approved by the Review Board of Puai Hospital of Tongji Medical College, Huazhong University of Science and Technology.

After 1 week acclimatization, male 15 -week old rats with stroke surgery were randomly allocated the following groups: (1) WKY: sham control $(n=16)$; (2) SHRsp: hypertensive sham (SHRSP alone, $n=16$ ); (3) SHRSP undergoing transient middle cerebral artery occlusion (tMCAO) for $60 \mathrm{~min}$ and receiving vehicle (SHRSP + tMCAO, $n=16$ ); (4) SHRSP undergoing tMCAO and treated with D-limonene (SHRSP + tMCAO + D-limonene, $\mathrm{n}=16) .1 \mathrm{~h}$ after the induction of tMCAO, animals were treated with $20 \mathrm{mg} / \mathrm{kg}$ of D-limonene (97\%; Sigma-Aldrich, St. Louis, MO, USA) intraperitoneally once daily for 14 days. The dose of D-limonene used in the study was selected according to our preliminary results and previous studies $(25,26)$. We have observed mild liver toxicity of $50 \mathrm{mg} / \mathrm{kg}$ D-limonene in mice.

Transient cerebral ischemia induced by occlusion of the right middle cerebral artery (MCA) was performed as previous described $(27,28)$. A midline incision was cut on the ventral surface of the neck to expose the right common carotid arteries which were ligated with 6.0 silk suture. The internal carotid and pterygopalatine artery were temporarily occluded for 60 min using a microvascular cliRelative cerebral blood flow (CBF) was monitored during the process of occlusion. After the 60 min of tMCAO, reperfusion was allowed by withdrawing the occluding filament back into the common carotid artery. Relative CBF was then monitored for $5 \mathrm{~min}$ and then the wound was sutured and the rats were then permitted to recover from anesthesia. Drug treatment was performed $5 \mathrm{~min}$ before the reperfusion. Sham-operated WKY and SHRSP rats were subjected only to exposure of the carotid arteries but not the occlusion.

Blood pressure measurement. Systolic blood pressure was determined in quiet conditions by means of the tail-cuff method after warming up the apparatus platform to $37^{\circ} \mathrm{C}$ for $10 \mathrm{~min}$. Each rat was introduced into a restrainer and a blood pressure tail-cuff was affixed to the base of the tail.

Morris water maze test. The Morris water maze test was conducted to evaluate the memory and cognitive function in rats. The Morris water maze test was performed as previously. In brief, a circular pool $(1.3 \mathrm{~m}$ in diameter, $50 \mathrm{~cm}$ in depth) was filled with room-temperature water. $1 \mathrm{~cm}$ below the surface of the water, a transparent platform was placed in the southeastern quadrant. On the first day, the rats were put into the pool without the platform for $2 \mathrm{~min}$ to get used to the experimental environment. Over the next 4 days, the platform acquisition test was conducted 4 times per day. The rats were released from different starting points and allowed to swim for $60 \mathrm{~s}$ to search for the platform. The escape latency time was defined by the time spent to find the hidden platform by the rats and times longer than $60 \mathrm{~s}$ were recorded as $60 \mathrm{~s} .24 \mathrm{~h}$ later, the rats were released from a new start position in the pool without the platform, which was termed the probe trial. The amount of time spent in the southeastern quadrant was recorded.

Novel object recognition test. The rats were individually placed into a plastic box with two objects with different forms. The rats were allowed to freely move in the box for $5 \mathrm{~min}$. This adaptive training was conducted again after $180 \mathrm{~min}$. In the short-term memory test, we replace one of the objects $180 \mathrm{~min}$ after the second training. The time spent by each animal with the novel object and the familiar object was recorded during a $10 \mathrm{~min}$ test period. In the long-term memory test, we replace the novel object $24 \mathrm{~h}$ after the second training. The time spent by each rat with the novel object and the familiar object was again recorded during a $10 \mathrm{~min}$ test period. In the tests, exploration was defined as sniffing or touching the object with the nose or foreleg. the objects were washed after each test to to avoid olfactory perception by the next rat.

\section{Behavioral tests}

Grip strength. The degree of force necessary to make the animal release a pull grid assembly with its forepaw was evaluated using a grip strength meter (Columbus Instruments, Columbus, OH, USA) as previously described (28). For each rat, a digital reading (in Newtons) of two successive trials was recorded and analyzed.

Somatosensory neglect test. The sensory impairments were detected by measurement of the detection of, and reaction to, small pieces of adhesive tape applied to the forelimbs after MCAO. We place a removable sticky tape on the ventral side of the animal's paw contralateral to the induced stroke. The time spent by each rat to contact or sense the tape and the time spent to remove it was recorded for $180 \mathrm{~s}$. Two successful trials for each animal were recorded for averaged analysis.

Spontaneous locomotor activity. Spontaneous motor activity was examined by Digiscan activity-monitoring boxes (AccuScan Instruments, Columbus, OH, USA). Each test was performed under red-light condition and lasted $5 \mathrm{~min}$.

Measurement of infarct volume. Rats were deeply anesthetized using isoflurane. Transcardial perfusion was conducted using cold saline followed by $10 \%$ buffered formalin. Brains were collected and then fixed in gradient sucrose solution, and cut coronally into $20-\mu \mathrm{m}$ sections as previously described (29).

Quantitative real-time PCR. Total RNA was extracted from brains using a RNA isolation kit and then reversely 
A

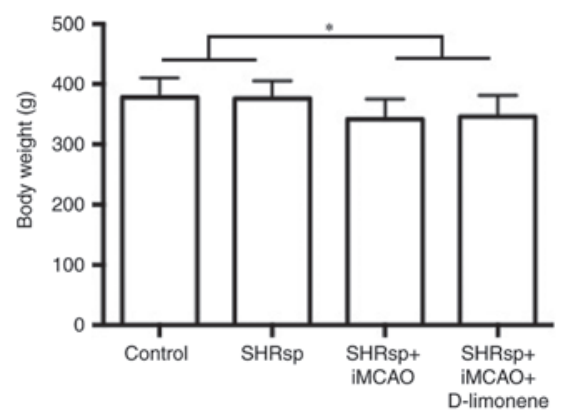

B

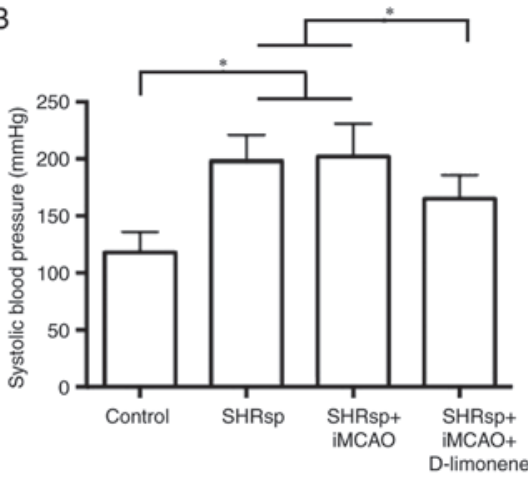

Figure 1. The effect of D-Limonene on systolic blood pressure in SHRsp rats after stroke. (A) Body weight (n=16). (B) The systolic blood pressure of the rats in each group was measured using the tail cuff method $(n=16)$. ${ }^{*}<<0.05$, between the groups.

transcribed into cDNA using an miScript II RT Kit (Thermo Fisher Scientific Inc., Waltham, MA, USA), according to the manufacturer's instructions. cDNA was used as a template in PCR reactions using gene-specific primer pairs. Real-time PCR was performed using an miScript SYBR Green PCR Kit (Thermo Fisher Scientific Inc., Waltham, MA, USA). $\beta$-actin was used as endogenous control. The fold changes of target gene expression were determined with the $\Delta \Delta \mathrm{Ct}$ method, and the relative expression of these genes was calculated by the $2^{-\Delta \mathrm{CT}}$ method and normalized to $\beta$-actin. IL-1 $\beta$ forward primer: 5'-CAGGCTTCGAGATGAACAACAA-3', and reverse primer: 5'-GTCCATTGAGGTGGAGAGCTTT-3'. MCP-1 forward primer: 5'-CTGTCACGCTTCTGGGCC TGT-3', and reverse primer: 5'-GCAGCAGGTGAGTGGGGC AT-3'. COX-2 forward primer: 5'-CCAGAGCAGAGAGAT GAAATACCA-3', and reverse primer: 5'-GCAGGGCGGGAT ACAGTTC-3'. VEGF forward primer: 5'-GATGAGATAGAG TATATCTTCAAGCCGT-3', and reverse primer: 5'-TCTATC TTTCTTTGGTCTGCATTCAC-3'. $\beta$-actin forward primer, 5'CTCCTGCTTGCTGATCCACATC-3', and reverse primer: 5'-CCACTGCCGCATCCTCTT-3'.

Oxidative stress. Superoxide dismutase (SOD) and catalase (CAT) and the levels of glutathione (GSH) and malondialdehyde (MDA) were determined using assay kits purchased from Nanjing Jiancheng Company (Jiangsu, China) as per the manufacturer's protocols. $20-\mu \mathrm{m}$ thick frozen brain sections were incubated with dihydroethidium (DHE) $(10 \mu \mathrm{M})$ (Invitrogen, Carlsbad, CA) in PBS for $30 \mathrm{~min}$ at $37^{\circ} \mathrm{C}$ in a humidified chamber in dark environment. The DHE-positive cells were observed under a fluorescence microscope and the positive cells were counted using with Image $\mathrm{J}$ software and expressed as percentage of control.

Statistical analysis. Data were expressed as mean \pm SEM. Data was analyzed using Graph Pad Prism software by one-way analysis of variance followed by a Turkey test for multiple comparisons. $\mathrm{P}<0.05$ was considered to be statistically significant.

\section{Results}

D-Limonene reduces blood pressure in SHRsp rats after stroke. Body weights were not significantly different between WKY and SHRsp rats (Fig. 1A). Stroke resulted in a marked decrease of body weight (Fig. 1A). D-Limonene did not significantly alter the body in SHRsp rats after stroke (Fig. 1A). The systolic blood pressure in SHRsp rats was significantly higher than that in WKY rats (Fig. 1B). Stroke did not result in a remarkable increase of systolic blood pressure in SHRsp rats (Fig. 1B). D-Limonene protected against the increase of systolic blood pressure SHRsp rats after stroke (Fig. 1B).

D-Limonene attenuates memory and cognitive impairment in SHRsp rats after stroke. The function of memory and cognition was evaluated by means of Morris water maze test and novel object recognition test. On the first day, no significant difference was observed in the hidden platform acquisition test among different groups. On the 3-5 days, the escape latency time in SHRsp rats was remarkably longer, compared with the WKY rats (Fig. 2A-E). Stroke resulted in a significant increase of escape latency time in SHRsp rats (Fig. 2A-E). The administration of D-Limonene markedly decreased the escape latency time in SHRsp rats after stroke (Fig. 2A-E). SHRsp rats spent less time in the target quadrant in the probe trial than that in WKY rats (Fig. 2F). Stroke resulted in a significant decrease of time spent in the target quadrant in the probe trial in SHRsp rats (Fig. 2F). The administration of D-Limonene markedly increased the time spent in the target quadrant in the probe trial in SHRsp rats after stroke (Fig. 2F).

In the novel object recognition tests, there was a marked decrease in the capacity to distinguish between familiar objects and novel objects in the SHRsp rats, compared with that in WKY rats (Fig. 2G and $\mathrm{H}$ ). Stroke resulted in a significant decrease of in the capacity to distinguish between familiar objects and novel objects in the SHRsp rats (Fig. 2G and H). The administration of D-Limonene markedly increased the capacity to distinguish between familiar objects and novel objects in the SHRsp rats in the short and long term memory tests (Fig. 2G and H).

D-Limonene attenuates behavioral impairment and infarct size in SHRsp rats after stroke. The behavioral tests were performed to evaluate the effect of D-Limonene on behavioral impairment in SHRsp rats after stroke. Grip strength in SHRsp rats was lower than that in WKY rats (Fig. 3A). Stroke resulted in a significant decrease of grip strength in the SHRsp 


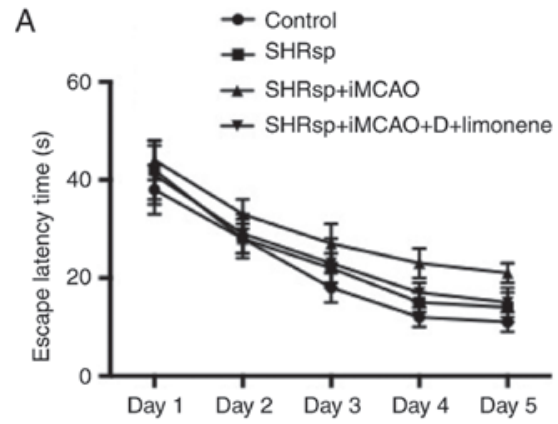

B

$$
\text { C }
$$

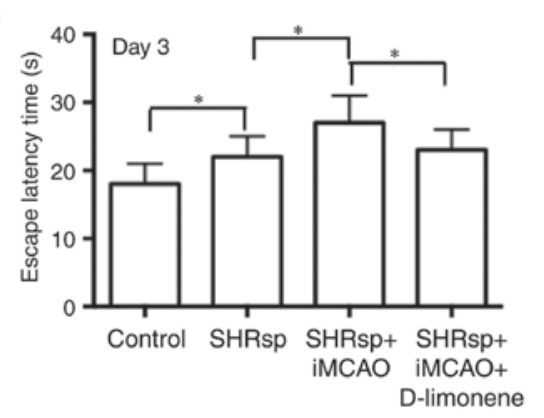

E
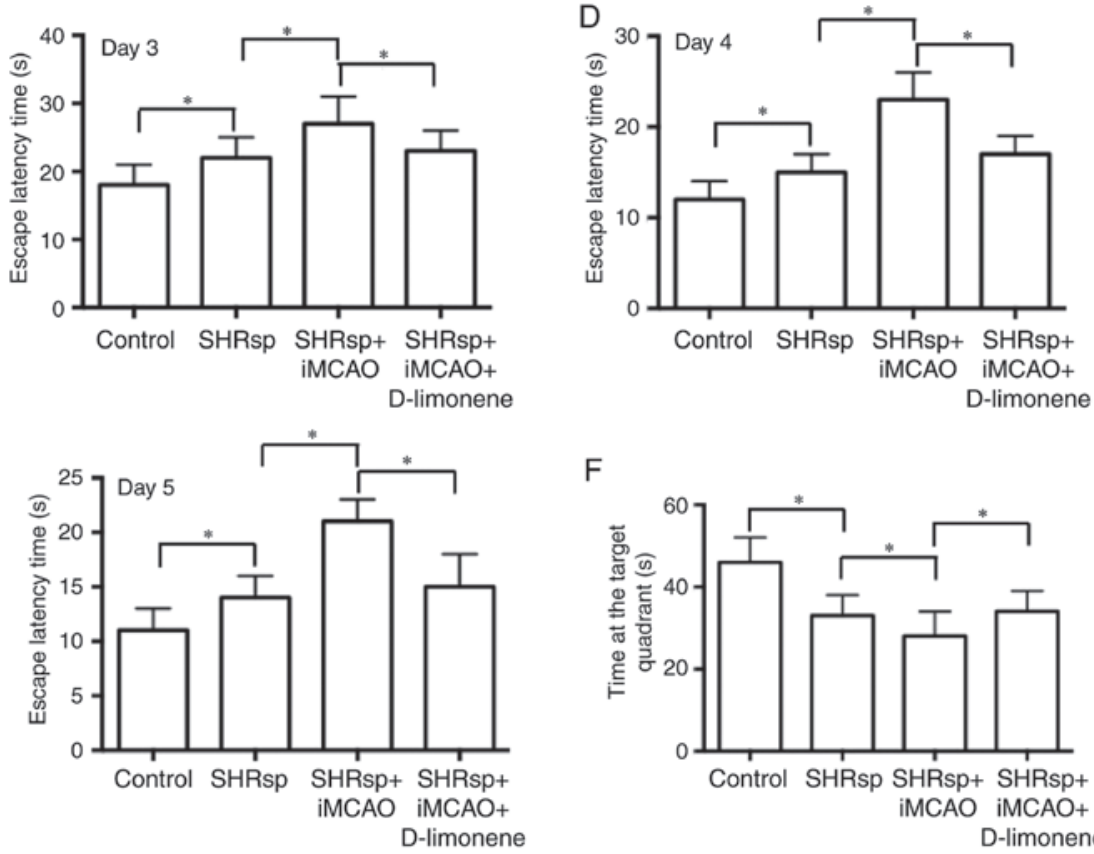

$\mathrm{F}$
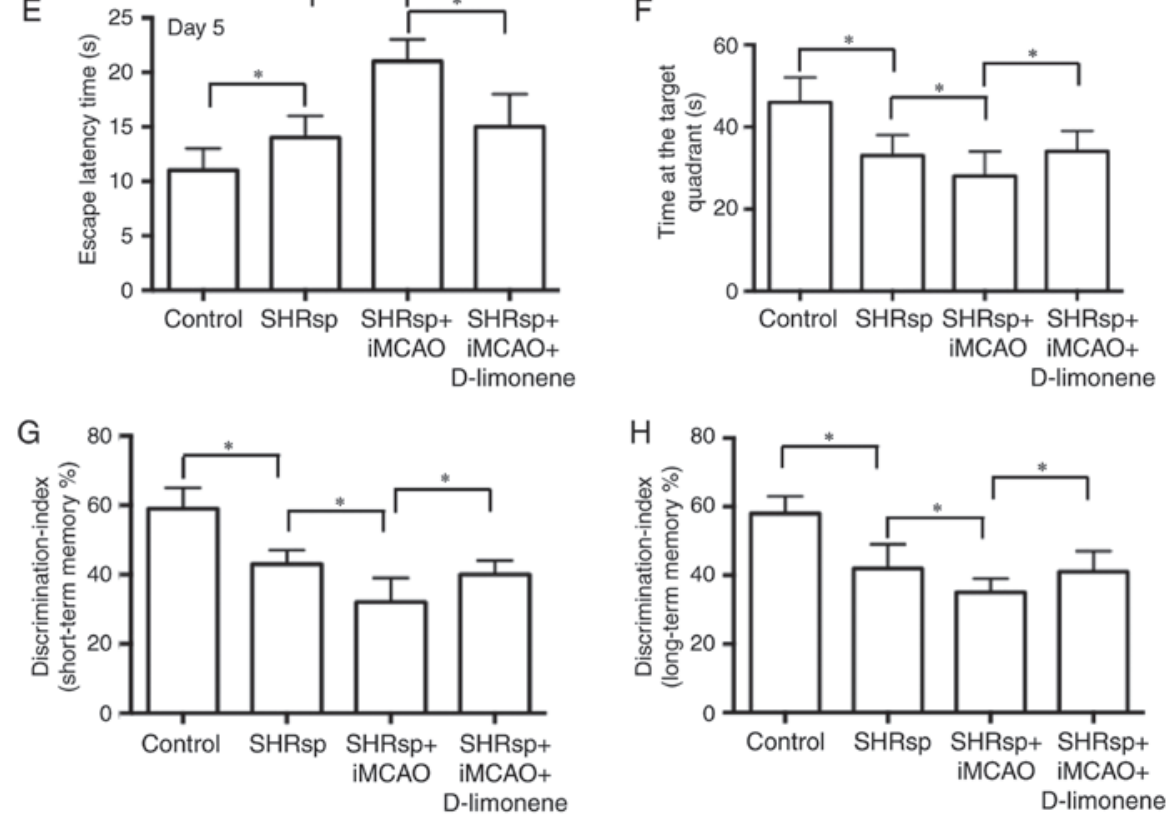

Figure 2. The effect of D-Limonene on memory and cognitive function in SHRsp rats after stroke. Morris water maze test and novel object recognition test were performed to evaluate memory and cognitive function. The escape latency time (n=8) was presented as (A) an overall value, and at (B) day 2 , (C) day 3 , (D) day 4 and (E) day 5. (F) Time spent at the target quadrant $(\mathrm{n}=8)$. (G) Discrimination-index in the short term memory test $(\mathrm{n}=8)$. (H) Discrimination-index in the long term memory test $(n=8)$. ${ }^{*}<<0.05$, between the groups.

rats (Fig. 3A). The administration of D-Limonene markedly increased grip strength in SHRsp rats after stroke (Fig. 3A). There was no significant difference of sensory neglect between SHRsp and WKY rats (Fig. 3B). Stroke resulted in a significant increase of sensory neglect in the SHRsp rats (Fig. 3B). The administration of D-Limonene markedly decreased sensory neglect in SHRsp rats after stroke (Fig. 3B). In the test of spontaneous motor activity, the distance travelled by SHRsp rats was significantly increased (Fig. 3C). However, stroke and D-Limonene had no significant effect on the distance travelled by the rats in SHRsp group (Fig. 3C). Ischemia-induced infarct volume in SHRsp rats was evaluated. As shown in Fig. 3D, the infarct volume induced by ischemia was significantly reduced by D-Limonene administration.

D-Limonene attenuates inflammation and increases angiogenesis in SHRsp rats after stroke. As shown in Fig. 4A-C, the mRNA expression of IL-1 $\beta$, monocyte chemoattractant protein-1 (MCP-1) and cyclooxygenase-2 (COX-2) was significantly higher in the brain of SHRsp rats compared with that of WKY rats. Stroke resulted in a significant increase in the mRNA expression of IL-1 $\beta$, MCP-1 and COX-2 in the SHRsp rats (Fig. 4A-C). The 
A

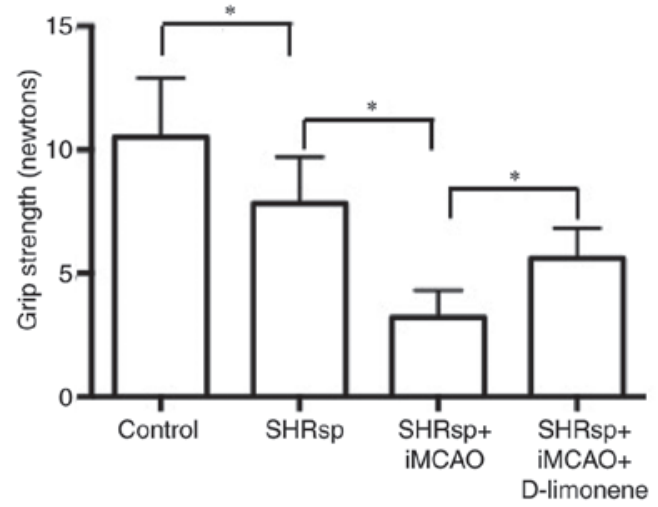

C

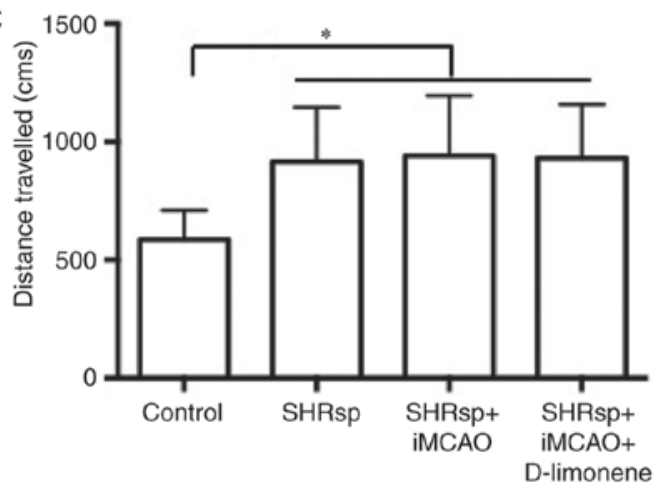

B

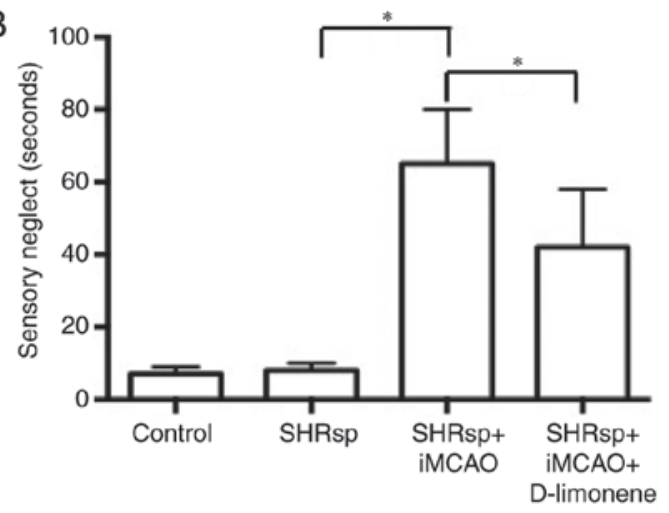

D 150

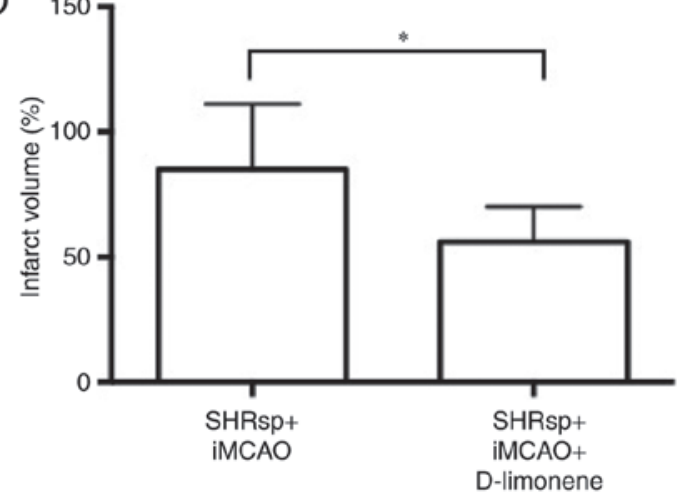

Figure 3. The effect of D-Limonene on behavioral function in SHRsp rats after stroke. (A) Grip strength (n=8) (B) Sensory neglect test ( $\mathrm{n}=8)$, (C) Locomotor activity $(\mathrm{n}=8)$ and (D) Infarction volume $(\mathrm{n}=8) .{ }^{*} \mathrm{P}<0.05$, between the groups.

A
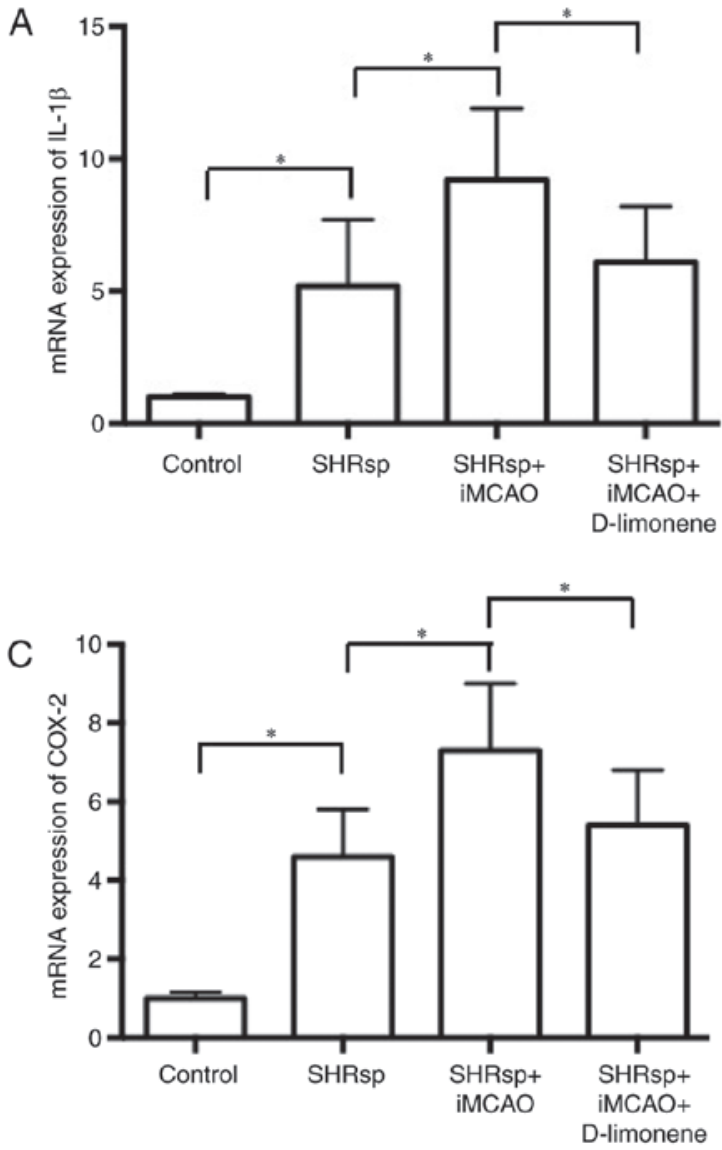

B
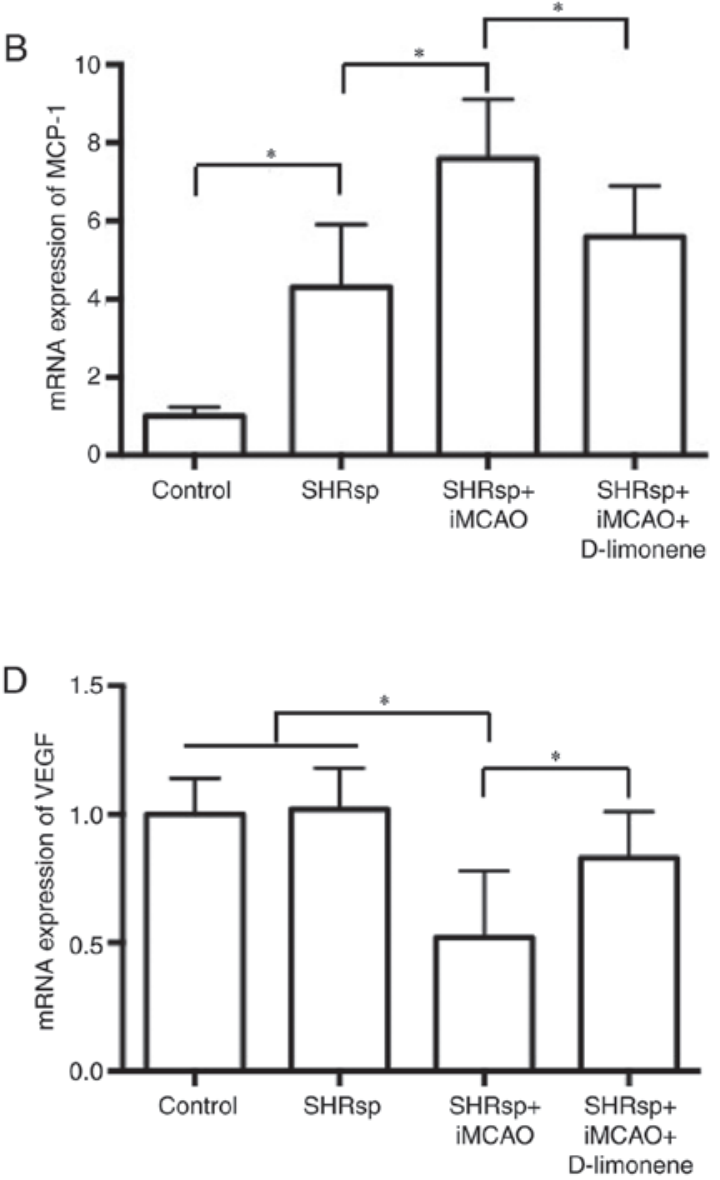

Figure 4. The effect of D-Limonene on inflammation and angiogenesis in SHRsp rats after stroke. mRNA expression of IL-1 $\beta$ (A), MCP-1 (B) and COX-2 (C), and VEGF (D) in the brain in SHRsp rats after stroke was determined by real-time PCR. (n=6). * $\mathrm{P}<0.05$, between the groups. 

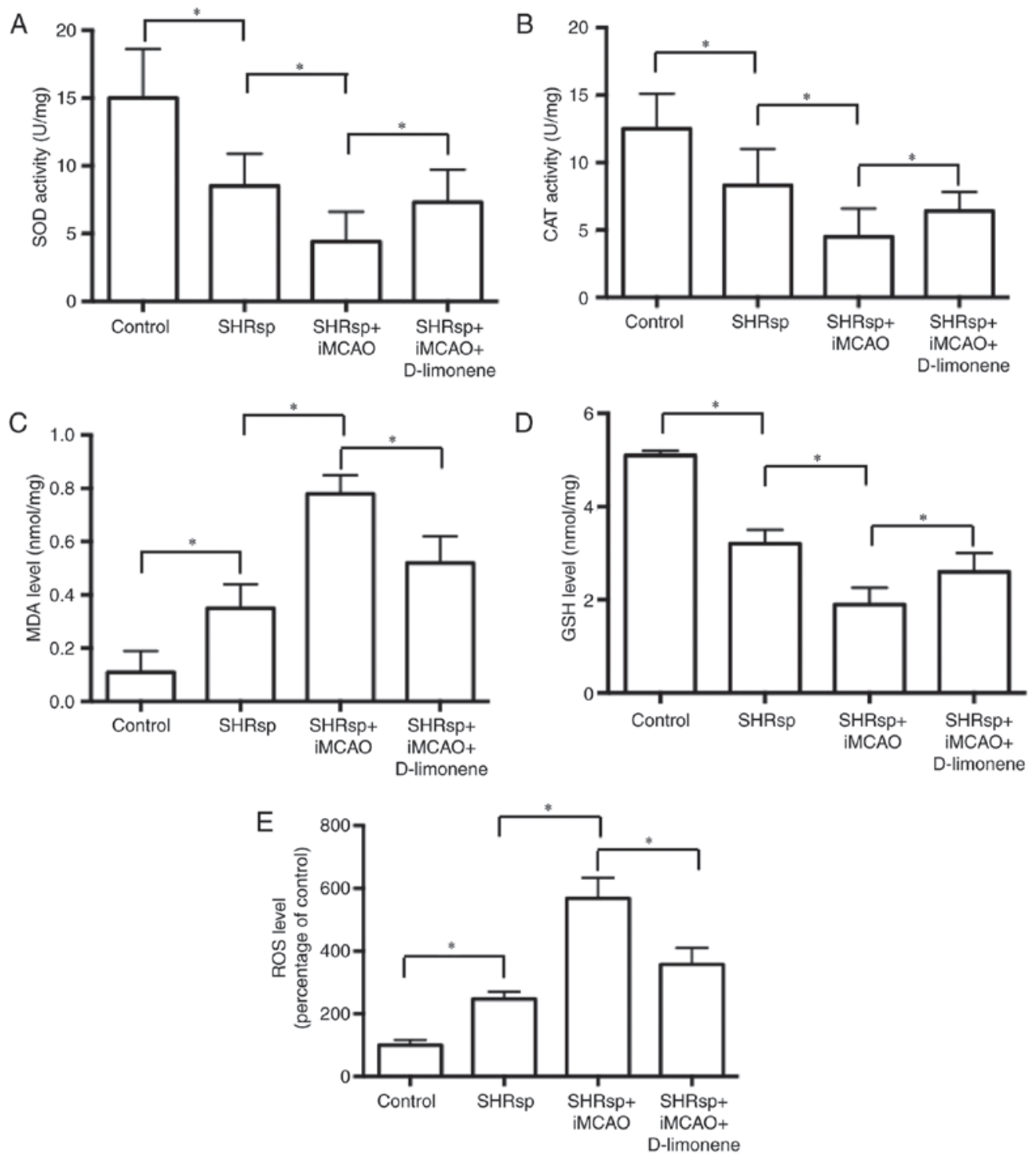

Figure 5. The effect of D-Limonene on oxidative stress in the brain in SHRsp rats after stroke. The activities of (A) SOD and (B) CAT, and levels of (C) MDA and (D) GSH were detected using commercial assay kits. (E) ROS level was determined by DHE staining and shown as percentage of control. (n=8). "P<0.05, between the groups.

administration of D-Limonene markedly decreased the mRNA expression of IL-1 $\beta$, MCP-1 and COX-2 in SHRsp rats after stroke (Fig. 4A-C). As shown in Fig. 4D, the mRNA expression of vascular endothelial growth factor (VEGF) was significantly decreased in the brain of SHRsp rats compared with that of WKY rats. Stroke resulted in a significant decrease in the mRNA expression of VEGF in the SHRsp rats (Fig. 4D). The administration of D-Limonene markedly increased the mRNA expression of VEGF in SHRsp rats after stroke (Fig. 4D).

D-Limonene attenuates oxidative stress in SHRsp rats after stroke. The effect of D-Limonene on oxidative stress under stroke condition was determined. As shown in Fig. 5, in SHRsp rats, the activities of antioxidant enzymes, including SOD (A) and CAT (B), was decreased, the level of MDA (C) was increased, the level of GSH (D) was decreased, and DHE-staining (E) was increased. Stroke resulted in a decrease of the activities of SOD and CAT, an increase of MDA, a decrease of GSH and an increase of DHE-staining (Fig. 5).
The administration of D-Limonene markedly increased the activities of SOD and CAT, decreased the levels of MDA, increased the GSH level and decreased the DHE-staining in SHRsp rats after stroke (Fig. 5).

\section{Discussion}

Previous studies have showed that D-Limonene can reduce monocrotaline-induced pulmonary hypertension (30). Natural products containing D-Limonene have been shown to attenuate stress-induced hypertension and stress responses in SHRsp rats $(23,24)$. In our study, we examined the effect of D-Limonene on changes of hypertension and ischemia-resulted neurological and vascular impairment in SHRsp rats. We showed that although the systolic blood pressure was not altered by ischemia operation, D-Limonene significantly decreased the systolic blood pressure in SHRsp rats after stroke. The administration of D-Limonene notably decreased the escape latency time and increased the time spent in the 
target quadrant in the probe trial in Morris water maze test, and markedly increased the capacity to distinguish between familiar objects and novel objects in the SHRsp rats in the short and long term memory tests. The results suggested that D-Limonene protected against ischemia-induced memory and cognitive impairment under the condition of hypertension.

It has been shown that D-Limonene may inhibit stimulant-induced behavioral changes via regulating dopamine levels and 5-HT receptor function (31). In the current study, we also evaluated the effect of D-Limonene on behavioral changes in SHRsp rats after stroke. We found that the administration of D-Limonene markedly increased grip strength and decreased sensory neglect in SHRsp rats after stroke, indicating that D-Limonene protected against ischemia-induced behavioral impairment under the condition of hypertension. The improvement of functional deficits could be attributed to the attenuation of infarct size after stroke.

Although the exact mechanisms and signaling pathways not clear, cerebral inflammation is implicated in the pathogenesis of hypertension and stroke. After transient cerebral ischemia, macrophages could be activated and produced IL-1 $\beta$, which is an important mediator of neurodegeneration. Increase of cytokines and regulators play critical roles in cerebral vascular damage and stroke injury. In the study, we found that D-Limonene inhibited the increase of IL-1 $\beta$, MCP-1 and COX-2 in SHRsp rats after stroke, indicating the amelioration of cerebral inflammation.

Vascular remodeling after stroke is an important process for neural regeneration $(32,33)$. Under the condition of hypertension, VEGF expression is reduced, resulting in reduction of nitric oxide production and leading to vascular constriction and reduction in sodium ion renal excretion (34). We found that stroke-resulted reduction of VEGF expression was significantly inhibited by D-Limonene. We speculated that D-Limonene may promote vascular remodeling through upregulation of VEGF expression.

SHRsp rats can produce more hydroxyl radicals than WKY rats, making them more vulnerable to oxidant damage after transient ischemic stroke (9). Attenuation of ROS levels has been shown to promote hypertensive cerebral vessel remodeling (35). Increase of ROS production, resulting in oxidative stress and playing critical roles in a variety of brain and neurological diseases. Previous studies have shown that D-Limonene have potential antioxidant activities under different conditions. In the current study, we also found that D-Limonene exhibited potent antioxidant effects through increasing SOD and CAT activities, decreasing MDA levels, increasing GSH levels and decreasing ROS levels. The results suggested that the antioxidant role of D-Limonene may be involved in the protective effects against neurological and vascular impairment in SHRsp rats after stroke.

In conclusion, we found that D-Limonene decreased blood pressure in ischemia-treated SHRsp rats and protected against memory and cognitive and behavioral impairment in SHRsp rats after stroke. Inhibition of cerebral inflammation, vascular remodeling and antioxidant activities of D-Limonene may be involved in the protective effects against ischemia-induced damage in SHRsp rats. In future studies, more attention should be paid to explore the molecular mechanism and find the target of D-Limonene. Overall, our study identified D-Limonene as a potential therapeutic candidate for treatment of stroke-resulted cerebral and vascular damage under the condition of hypertension.

\section{References}

1. Pantoni L: Cerebral small vessel disease: From pathogenesis and clinical characteristics to therapeutic challenges. Lancet Neurol 9: 689-701, 2010.

2. Debette $S$ and Markus HS: The clinical importance of white matter hyperintensities on brain magnetic resonance imaging: Systematic review and meta-analysis. BMJ 341: c3666, 2010.

3. Madigan JB, Wilcock DM and Hainsworth AH: Vascular contributions to cognitive impairment and dementia: Topical review of animal models. Stroke 47: 1953-1959, 2016.

4. Jokinen H, Kalska H, Ylikoski R, Madureira S, Verdelho A, van der Flier WM, Scheltens P, Barkhof F, Visser MC, Fazekas F, et al: Longitudinal cognitive decline in subcortical ischemic vascular disease-the LADIS Study. Cerebrovasc Dis 27: 384-391, 2009.

5. Gouw AA, van der Flier WM, Pantoni L, Inzitari D, Erkinjuntti T, Wahlund LO, Waldemar G, Schmidt R, Fazekas F, Scheltens P, et al: On the etiology of incident brain lacunes: Longitudinal observations from the LADIS study. Stroke 39: 3083-3085, 2008.

6. Centers for Disease Control and Prevention (CDC): Prevalence of stroke-United States, 2006-2010. MMWR Morb Mortal Wkly Rep 61: 379-82, 2012.

7. Yamori Y: Importance of genetic factors in stroke: An evidence obtained by selective breeding of stroke-prone and -resistant SHR. Jpn Circ J 38: 1095-1000, 1974.

8. Rubattu S, Lee-Kirsch MA, DePaolis P, Giliberti R, Gigante B, Lombardi A, Volpe M and Lindpaintner K: Altered structure, regulation, and function of the gene encoding the atrial natriuretic peptide in the stroke-prone spontaneously hypertensive rat. Circ Res 85: 900-905, 1999.

9. Yamagata K, Kitazawa T, Shinoda M, Tagawa C, Chino M and Matsufuji H: Stroke status evoked adhesion molecule genetic alterations in astrocytes isolated from stroke-prone spontaneously hypertensive rats and the apigenin inhibition of their expression. Stroke Res Treat 2010: pii: 386389, 2010.

10. Tagami M, Ikeda K, Yamagata K, Nara Y, Fujino H, Kubota A, Numano F and Yamori Y: Vitamin E prevents apoptosis in hippocampal neurons caused by cerebral ischemia and reperfusion in stroke-prone spontaneously hypertensive rats. Lab Invest 79: 609-615, 1999.

11. Mărgăritescu $\mathrm{O}$, Pirici D and Mărgăritescu C: VEGF expression in human brain tissue after acute ischemic stroke. Rom J Morphol Embryol 52: 1283-1292, 2011.

12. Banthorpe DV, Charlwood BV and Francis MJ: The biosynthesis of monoterpenes. Chem Rev 72: 115-155, 1972

13. Wattenberg LW: Inhibition of carcinogenesis by minor anutrient constituents of the diet. Proc Nutr Soc 49: 173-183, 1990.

14. Marshall JR: Improving Americans' diet-setting public policy with limited knowledge. Am J Public Health 85: 1609-1611, 1995.

15. National Toxicology Program: NTP Toxicology and Carcinogenesis Studies of d-Limonene (CAS No. 5989-27-5) in F344/N Rats and B6C3F1 Mice (Gavage Studies). Natl Toxicol Program Tech Rep Ser 347: 1-165, 1990.

16. Chaudhary SC, Siddiqui MS, Athar M and Alam MS: D-Limonene modulates inflammation, oxidative stress and Ras-ERK pathway to inhibit murine skin tumorigenesis. Hum Exp Toxicol 31: 798-811, 2012.

17. Tounsi MS, Wannes WA, Ouerghemmi I, Jegham S, Ben Njima Y, Hamdaoui G, Zemni H and Marzouk B: Juice components and antioxidant capacity of four Tunisian Citrus varieties. J Sci Food Agric 91: 142-151, 2011.

18. Roberto D, Micucci P, Sebastian T, Graciela F and Anesini C: Antioxidant activity of limonene on normal murine lymphocytes: Relation to $\mathrm{H} 2 \mathrm{O} 2$ modulation and cell proliferation. Basic Clin Pharmacol Toxicol 106: 38-44, 2010.

19. Lu XG, Zhan LB, Feng BA, Qu MY, Yu LH and Xie JH: Inhibition of growth and metastasis of human gastric cancer implanted in nude mice by d-limonene. World J Gastroenterol 10: 2140-2144, 2004.

20. Manuele MG, Barreiro Arcos ML, Davicino R, Ferraro G, Cremaschi G and Anesini C: Limonene exerts antiproliferative effects and increases nitric oxide levels on a lymphoma cell line by dual mechanism of the ERK pathway: Relationship with oxidative stress. Cancer Invest 28: 135-145, 2010. 
21. Bodake HB, Panicker KN, Kailaje VV and Rao KV: Chemopreventive effect of orange oil on the development of hepatic preneoplastic lesions induced by N-nitrosodiethylamine in rats: An ultrastructural study. Indian J Exp Biol 40: 245-251, 2002.

22. Del Toro-Arreola S, Flores-Torales E, Torres-Lozano C, Del Toro-Arreola A, Tostado-Pelayo K, Guadalupe Ramirez-Dueñas M and Daneri-Navarro A: Effect of D-limonene on immune response in $\mathrm{BALB} / \mathrm{c}$ mice with lymphoma. Int Immunopharmacol 5: 829-838, 2005.

23. Shibata H, Fujiwara R, Iwamoto $M$, Matsuoka $H$ and Yokoyama MM: Immunological and behavioral effects of fragrance in mice. Int J Neurosci 57: 151-159, 1991.

24. Kawakami K, Kawamoto M, Nomura M, Otani H, Nabika T and Gonda T: Effects of phytoncides on blood pressure under restraint stress in SHRS. Clin Exp Pharmacol Physiol 31 (Suppl 2): S27-S28, 2004

25. Rehman MU, Tahir M, Khan AQ, Khan R, Oday-O-Hamiza, Lateef A, Hassan SK, Rashid S, Ali N, Zeeshan M and Sultana S: D-limonene suppresses doxorubicin-induced oxidative stress and inflammation via repression of COX-2, iNOS, and NFאB in kidneys of Wistar rats. Exp Biol Med (Maywood) 239: 465-476, 2014

26. Victor Antony Santiago J, Jayachitra J, Shenbagam M and Nalini N: Dietary d-limonene alleviates insulin resistance and oxidative stress-induced liver injury in high-fat diet and L-NAME-treated rats. Eur J Nutr 51: 57-68, 2012.

27. Longa EZ, Weinstein PR, Carlson S and Cummins R: Reversible middle cerebral artery occlusion without craniectomy in rats. Stroke 20: 84-91, 1989

28. Yousuf S, Atif F, Sayeed I, Wang J and Stein DG: Neuroprotection by progesterone after transient cerebral ischemia in stroke-prone spontaneously hypertensive rats. Horm Behav 84: 29-40, 2016.
29. Yousuf S, Sayeed I, Atif F, Tang H, Wang J and Stein DG: Delayed progesterone treatment reduces brain infarction and improves functional outcomes after ischemic stroke: A time-window study in middle-aged rats. J Cereb Blood Flow Metab 34: 297-306, 2014.

30. Touvay C, Vilain B, Carré C, Mencia-Huerta JM and Braquet $\mathrm{P}$ Effect of limonene and sobrerol on monocrotaline-induced lung alterations and pulmonary hypertension. Int Arch Allergy Immunol 107: 272-274, 1995.

31. Yun J: Limonene inhibits methamphetamine-induced locomotor activity via regulation of 5-HT neuronal function and dopamine release. Phytomedicine 21: 883-887, 2014

32. Li Z, Wang B, Kan Z, Zhang B, Yang Z, Chen J, Wang D, Wei H, Zhang JN and Jiang R: Progesterone increases circulating endothelial progenitor cells and induces neural regeneration after traumatic brain injury in aged rats. J Neurotrauma 29: 343-353, 2012.

33. Liu J, Wang Y, Akamatsu Y, Lee CC, Stetler RA, Lawton MT and Yang GY: Vascular remodeling after ischemic stroke: Mechanisms and therapeutic potentials. Prog Neurobiol 115: 138-156, 2014.

34. Lankhorst S, Saleh L, Danser AJ and van den Meiracker AH Etiology of angiogenesis inhibition-related hypertension. Curr Opin Pharmacol 21: 7-13, 2015.

35. Iadecola C and Davisson RL: Hypertension and cerebrovascular dysfunction. Cell Metab 7: 476-484, 2008.

(i) (8) This work is licensed under a Creative Commons Attribution-NonCommercial-NoDerivatives 4.0 International (CC BY-NC-ND 4.0) License. 